\title{
Satellite-enabled LTE systems in LEO Constellations
}

\author{
A. Guidotti*, A. Vanelli-Coralli*, M. Caus ${ }^{\dagger}$, J. Bas ${ }^{\dagger}$, G. Colavolpe ${ }^{\ddagger}$, T. Foggi ${ }^{\ddagger}$, S. Cioni $^{\S}$, A. Modenini ${ }^{\S}$, D. Tarchi $^{*}$ \\ ${ }^{*}$ Dept. of Electrical, Electronic, and Information Engineering (DEI), Univ. of Bologna, Bologna, Italy \\ Email: \{a.guidotti,alessandro.vanelli,daniele.tarchi\}@ unibo.it \\ ${ }^{\dagger}$ Centre Tecnològic de Telecomunicacions de Catalunya, Castelldefels, Barcelona, Spain \\ Email: \{marius.caus,joan.bas\}@cttc.es \\ $\ddagger$ Dept. of Information Engineering, Univ. of Parma, Parma, Italy \\ Email: giulio.colavolpe@unipr.it,tommaso.foggi@nemo.unipr.it \\ $\S$ European Space Agency - esa.int, ESTEC/ETC, The Netherlands \\ Email: stefano.cioni@esa.int
}

\begin{abstract}
LTE-based satellite systems in LEO constellations are a promising solution for extending broadband coverage to areas not connected to a terrestrial infrastructure. However, the large delays and Doppler shifts over the satellite channel pose severe technical challenges to a traditional LTE system. In this paper, two architectures are proposed for a LEO megaconstellation realizing a satellite-enabled LTE system, in which the on-ground LTE entity is either an eNB (Sat-eNB) or a Relay Node (Sat-RN). Focusing on the latter, the impact of large delays and Doppler shifts on LTE PHY/MAC procedures is discussed and assessed. It will be shown that, while carrier spacings, Random Access, and RN attach procedures do not pose specific issues, HARQ requires substantial modifications. Moreover, advanced handover procedures will be also required due to the satellites' movement.
\end{abstract}

\section{INTRODUCTION}

Today, Long Term Evolution (LTE) Release 13, [1], is the latest cellular standard designed for providing broadband connectivity in terrestrial systems, reaching up to $100 \mathrm{Mbps}$ in downlink by means of high-speed backhaul infrastructures, e.g., through fiber-optic communications. However, due to the large deployment costs, in remote and rural areas traditional backhauling solutions are not profitable for network operators. Moreover, in emergency situations, the terrestrial infrastructures are often destroyed, thus totally isolating the area hit by a natural disaster or a terroristic attack when connectivity is most needed. In this context, Satellite Communications (SatCom) provide a valuable cost-effective opportunity. Thanks to their inherently large footprint, satellites can complement and extend terrestrial networks, not only in rural areas and emergency situations, but also for traffic off-loading of dense terrestrial networks.

Today, High Throughput Satellites (HTS) provide large capacity connectivity through frequency reuse and multi-spot beam technology at reduced costs. In addition, both Geostationary Earth Orbit (GEO) and non-GEO systems are deployed to serve air, sea, and remote land areas in L-, S-, and Ka-bands to mobile and fixed terminals and backhaul services are also available in $\mathrm{Ka}-/ \mathrm{Ku}$-bands, [2]. The integration of terrestrial systems with GEO satellites would thus provide an effective global coverage with high throughput, but the large delays and Doppler shifts when considering geostationary orbits pose challenging issues. In particular, in [3]-[5], the authors analyzed resource allocation for multicast transmissions and TCP protocol performance in a GEO LTE-based satellite system, providing valuable solutions. However, to circumvent the above issues, an ever increasing attention is being gained by Low Earth Orbit (LEO) systems and, in particular, by megaconstellations, i.e., systems in which hundreds of satellites are deployed, as demonstrated by several recent commercial endeavors.

In this paper, we consider a system in which a megaconstellation of LEO satellites is deployed in $\mathrm{Ku}$-band to provide LTE broadband services to areas not connected to a terrestrial infrastructure. Each satellite in the mega-constellation covers several satellite-enabled network entities that create onground LTE cells. Depending on the satellite-enabled LTE entity, two architectures are identified: i) Sat-RN, in which the on-ground cell is created through a LTE Relay Node (RN); and ii) Sat-eNB, in which a traditional eNB is considered. Focusing on the former, the impact of delays and Doppler shifts in LEO systems on both the LTE waveforms and PHY/MAC procedures is analyzed and several solutions are also proposed.

\section{SySTEM MODEL}

LEO satellites are deployed between 500 and $2000 \mathrm{~km}$ from Earth, thus allowing to extend and complement terrestrial networks with reduced path loss and delay when compared to GEO satellites. In Ku-band, significant signal degradation is experienced due to rain and cloud attenuation, which are even more pronounced in LEO systems because of the low elevation angles at which satellites are seen for a non-negligible percentage of time. However, the variability in the received signal is reduced, thus increasing the related throughput, through Adaptive Coding and Modulation (ACM).

In terms of system architecture, the following assumptions are made for the considered LEO mega-constellation system: i) the 


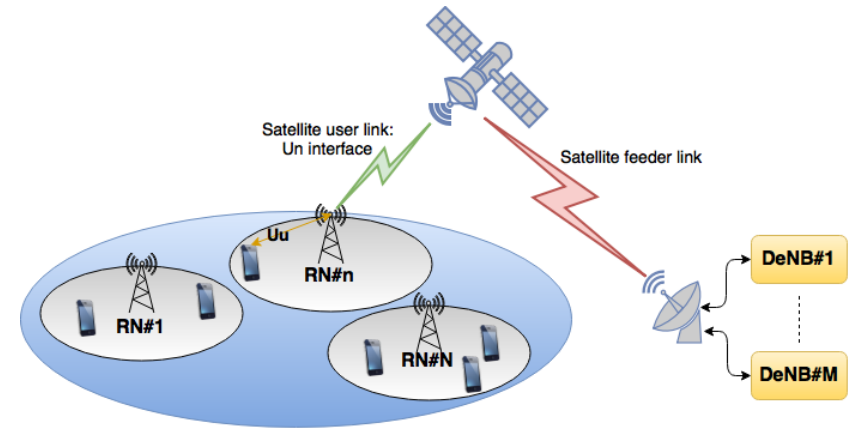

Fig. 1. Option A: Sat-RN architecture.

terrestrial terminals in each on-ground cell are assumed to be LTE User Equipments (UEs) connected to the satellite-enabled entity through a traditional Uu air interface; ii) the satellites are assumed to be transparent and to provide backhaul connection to the satellite-enabled entities; and iii) the satellite gateway (GW) is connected to the satellites through ideal satellite feeder links, providing access to the core network. Depending on the type of LTE satellite-enabled network entity, two different solutions, shown in Figs. 1-2 and described in the following, can be considered. Finally, we focus on the FDD framing structure for the LTE system, since TDD is not feasible due to the large delays in the considered system.

\section{A. Option A: Sat-RN}

In the first option, we assume that the satellite-enabled network entity is a satellite-enabled Relay Node (Sat-RN), as shown in Fig. 1, where the satellite user link connects the RN to a Donor eNB (DeNB), conceptually located at the system gateway, which interacts with the LTE Evolved Packet Core network (EPC). This option exploits the concept of LTE RN, a low-power base station wirelessly connected to a DeNB, introduced in Rel. 8, [1], [6]. Two important aspects are worth mentioning: i) on the backhaul link (RN-to-DeNB), the $\mathrm{RN}$ is connected to the DeNB through a modified Uu air interface, the Un air interface, while on the radio access link (UE-to$\mathrm{RN}$ ) the UEs are still connected to the $\mathrm{RN}$ through the $\mathrm{Uu}$ interface; and ii) the RN terminates the Uu, S1 (air interface between eNBs and EPC), and X2 (air interface between eNBs) radio protocols, i.e., up to Layer 3 . Thus, as a matter of facts, from the UE perspective the RN acts as an eNB, while from the DeNB point of view the $\mathrm{RN}$ is seen as a UE. Moreover, the Un interface is based on the same radio protocols and procedures used in the well-known $\mathrm{Uu}$ air interface and that the only differences are in the RF characteristics and minimum performance requirements, [1], [7]. Thus, for both the backhaul and access links, the system adopts Orthogonal Frequency Division Multiplexing (OFDM) in the downlink and Single-Carrier Frequency Division Multiple Access (SCFDMA) in the uplink. Finally, a single DeNB can manage more than one $\mathrm{RN}$, i.e., $M \leq N$ where $M$ and $N$ denote the number of DeNBs and RNs, respectively.

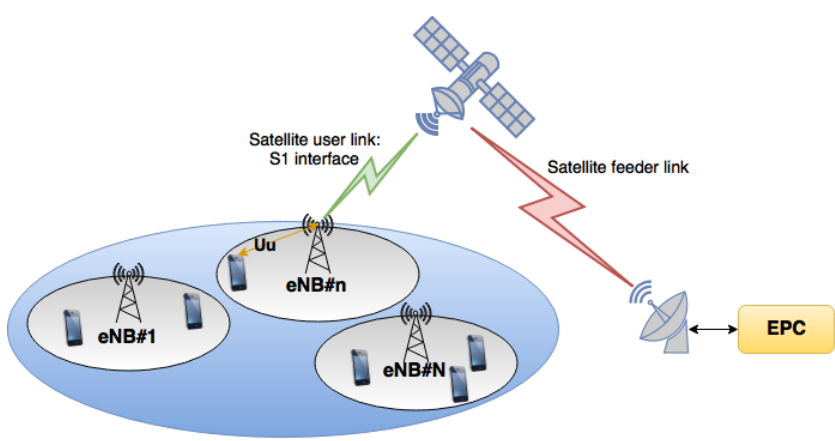

Fig. 2. Option B: Sat-eNB architecture.

\section{B. Option B: Sat-eNB}

In the second option, the satellite-enabled network entity is a satellite-enabled eNB (Sat-eNB), as in Fig. 2. Since we have a traditional eNB providing on-ground connectivity to the UEs, the satellite user link adopts the S1 interface. The S1 interface is an open interface, i.e., it can be any radio interface as long as a few mandatory requirements are met, [8], [9]. In particular, it shall support the exchange of signalling information between the eNB and EPC and facilitate the connection among eNBs from different manufacturers, and facilitate the introduction of future technologies. Thus, for Sat-eNB, the interface can be realized as a Uu, Un, or a SatCom specific-designed (e.g., DVB-S2X) air interface as long as the mandatory requirements for $\mathrm{S} 1$ interfaces are met.

\section{SATELlite CHANNEL}

The applicability of LTE in a satellite scenario is limited by the impact of the satellite channel impairments on its requirements and procedures. In particular, specific attention must be paid to the Round Trip Time (RTT) and the Doppler shift. The RTT is twice the propagation delay between the transmitter and the receiver, under the assumption that the propagation delay is the same for both uplink and downlink. The Doppler shift is the change in the carrier frequency due to the relative motion between the satellite and the user terminal. The maximum Doppler shift can be computed for a given satellite altitude and carrier frequency and minimum elevation angle at which the satellite is seen from the terminal [10]. In the following, the Doppler shift tolerated by the receiver as well as the impact of RTT on LTE protocols will be discussed. It shall be noticed that, in the considered scenario, we have both the access link (UE-to-RN) and the backhaul link (RN-toDeNB). However, only the backhaul link will be considered for such analysis, as the access link is involved in a traditional LTE cell for which no modification to procedures or requirements shall be introduced. Besides general considerations, we have assumed a satellite constellation operating in $\mathrm{Ku}$-band and characterized by an altitude $h=1200 \mathrm{~km}$, a beam size of approximately $320 \mathrm{~km}$, and a minimum elevation angle of $45^{\circ}$. 


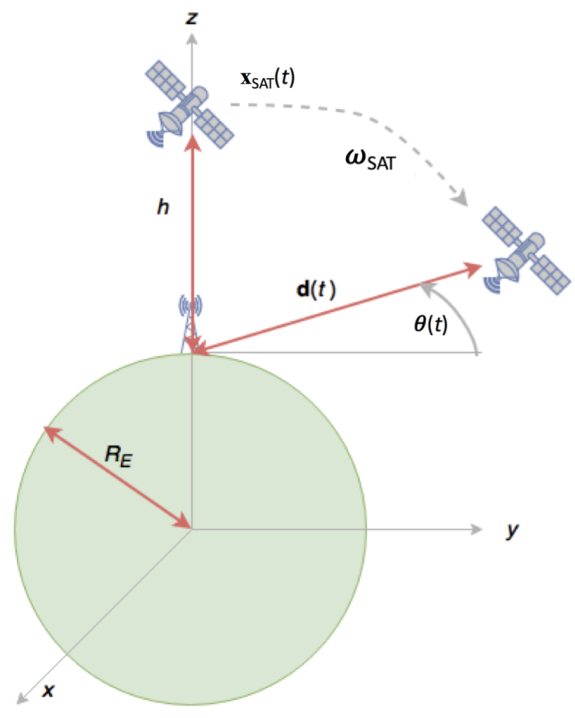

Fig. 3. Geometry for the computation of the Doppler shift.

\section{A. Delay}

The propagation delay is a critical issue, since it yields a misalignment between uplink and downlink frames and it also affects MAC layer protocols. This is especially relevant in SatCom, where distances are very large. In a traditional LTE cell, a UE far from the eNB experiences a larger propagation delay and, thus, its uplink transmission is received later by the eNB, with respect to a UE closer to the eNB. In order to cope with such time misalignment, LTE introduces a technique called Timing Advance (TA), which allows to adjust the transmission time in order to synchronize uplink and downlink frames at the eNB. Depending on the equipment configuration, the maximum delays in an LTE cell are included in the range $[133.33,688.021] \mu \mathrm{s},[11]$. The maximum timing advance allowed by the protocol is $T_{\mathrm{AD}}=0.6667 \mathrm{~ms}$, and is defined as $T_{\mathrm{AD}}=16 \times T_{\mathrm{A}} \times T_{s}$, where $T_{s}=1 /(2048 \times 15000)$ $\mathrm{s}$ is the sampling time and $T_{\mathrm{A}}$ is a parameter included in the range [0,1282], [12].

The propagation delay of a satellite in a LEO constellation is much larger than the maximum $T_{\mathrm{AD}}$ foreseen by the LTE standard. In fact, the RTT can be easily computed for the considered scenario, finding $T_{\mathrm{RTT}} \approx 16 \mathrm{~ms}$. However, no modification to the TA is required since: i) in the access link, we have a traditional LTE cell for which no modifications are needed; and ii) on the backhaul link, the RN gathers information from all of the UEs in its cell, aggregates the data, and transmits it to the satellite, which implies that all uplink transmissions are coming from the same entity. Although no modifications are needed to the TA, the large RTT in a satellite system will have an impact on the PHY and MAC layers, e.g., the RA as well as the HARQ retransmission scheme, as will be discussed in the following sections.

\section{B. Doppler}

In order to compute the maximum Doppler shift, LTE considers the case of high speed trains. In this scenario, it is assumed that the speed of the train is around $v=500 \mathrm{~km} / \mathrm{h}$ and that the carrier frequency is $f_{\mathrm{c}}=2 \mathrm{GHz}$. Thus, the maximum Doppler shift results in $f_{\mathrm{d}}=950 \mathrm{~Hz}$, and, according to the Nyquist sampling theorem, the maximum sampling period to correctly estimate the channel is $0.5 \mathrm{~ms}$.

In the proposed system, two cases have to be considered. On the one hand, for the access link (UE-to-RN), the situation is the same as for terrestrial LTE. On the other hand, on the backhaul link, i.e., when the $\mathrm{RN}$ is communicating with the DeNB through a LEO satellite, the Doppler shift will be much larger with respect to that considered in LTE specifications. In particular, the Doppler shift is equal to zero when the satellite is at the zenith, while at lower elevation angles we have larger values that have to be coped with. In order to compute the Doppler shift in the worst case, we refer to Fig. 3. The Doppler shift experienced by a generic RN can be computed as follows as a function of time:

$$
f_{\mathrm{d}}(t)=\frac{f_{0}}{c} \frac{\mathbf{d}(t)}{|\mathbf{d}(t)|} \frac{\partial \mathbf{x}_{\mathrm{SAT}}(t)}{\partial t}
$$

where $f_{0}$ is the carrier frequency, $\mathbf{d}(t)$ is the distance vector between the satellite and the RN, and $\mathbf{x}_{\mathrm{SAT}}(t)$ is the vector of the satellite position:

$$
\mathbf{x}_{\mathrm{SAT}}(t)=\left[0,\left(R_{\mathrm{E}}+h\right) \sin \left(\omega_{\mathrm{SAT}} t\right),\left(R_{\mathrm{E}}+h\right) \cos \left(\omega_{\mathrm{SAT}} t\right)\right]^{T}
$$

Referring to the geometry provided in Fig. 3, we have:

$$
\mathbf{d}(t)=\left[0,\left(R_{\mathrm{E}}+h\right) \sin \left(\omega_{\mathrm{SAT}} t\right),\left(R_{\mathrm{E}}+h\right) \cos \left(\omega_{\mathrm{SAT}} t\right)-R_{\mathrm{E}}\right]^{T}
$$

where $R_{E}$ is the Earth radius, $h$ is the satellite altitude, and $\omega_{S A T}$ is the satellite angular velocity. To express the Doppler shift as a function of the satellite elevation angle, we need to write $\omega_{S A T} t$ as a function of $|\mathbf{d}(t)|, \theta(t), h$, and $R_{E}$. Such relation can be easily obtained as follows:

$$
\cos \left(\omega_{\mathrm{SAT}} t\right)=\frac{R_{E}+|\mathbf{d}(t)| \sin \left(\omega_{\mathrm{SAT}} t\right)}{R_{E}+h} .
$$

By plugging (2), (3) and (4) into (1) we get

$$
\begin{gathered}
f_{\mathrm{d}}(t)=\frac{f_{0} R_{E} \omega_{S A T}}{c} \times \\
\sqrt{\frac{h^{2}+2 R_{E} h-2 R_{E}|\mathbf{d}(t)| \sin (\theta(t))-|\mathbf{d}(t)|^{2} \sin ^{2}(\theta(t))}{h^{2}+2 R_{E} h-2 R_{E}|\mathbf{d}(t)| \sin (\theta(t))}} .
\end{gathered}
$$

Recalling the law of cosines, we can relate the sides of the triangle defined by the center of the Earth, $\mathbf{x}_{\mathrm{SAT}}(t)$ and the RN position as

$$
\left(R_{E}+h\right)^{2}-R_{E}^{2}-|\mathbf{d}(t)|^{2}=2 R_{E}|\mathbf{d}(t)| \sin (\theta(t)) .
$$

By substituting (6) into (5), we finally obtain the following closed-form expression of the Doppler shift as a function of the elevation angle:

$$
f_{\mathrm{d}}(t)=\frac{f_{0} \omega_{\mathrm{SAT}} R_{\mathrm{E}} \cos (\theta(t))}{c}
$$


where $\omega_{\text {SAT }}=\sqrt{G M_{E} /\left(R_{E}+h\right)^{3}}, G=6,6710^{-11} \mathrm{~N}$. $\mathrm{m}^{2} / \mathrm{kg}^{2}$ is the Gravitational constant, and $M_{\mathrm{E}}=5,98 \cdot 10^{24}$ $\mathrm{kg}$ is Earth mass. In the considered scenario, it can be derived that the maximum Doppler shift is within the range $158 \mathrm{kHz}$ $\leq f_{\mathrm{d}} \leq 201 \mathrm{kHz}$, being the carrier frequency in Ku-band in the range $11 \mathrm{GHz} \leq f_{0} \leq 14 \mathrm{GHz}$. Thus, the Doppler shift in the satellite channel is increased by a factor $x$, with $166<x<211$, with respect to the maximum Doppler shift experienced in LTE.

\section{IMPACT ON LTE}

In this section, we discuss the impact of delay and Doppler shift on LTE MAC and PHY layers in the Sat-RN architecture. In fact, the Sat-eNB option has an intrinsic standard-compliant solution for an optimized waveform on the satellite link.

\section{A. Waveform}

As reported in Section II, the downlink (uplink) waveform is based on OFDM (SC-FDMA). The downlink (uplink) transmission resources are defined in three dimensions: time, frequency, and space. The latter is measured in layers and is accessed in terms of antenna ports at the eNB. Downlink and uplink transmissions are organised into frames with $10 \mathrm{~ms}$ duration, consisting of 20 time slots with duration $1 \mathrm{~ms}$. In each time slot there are 6 or 7 OFDM (SC-FDMA) symbols in the downlink (uplink), according to what type of cyclic prefix (CP) is being used. When an extended CP is implemented, the subcarrier spacing $\Delta f$ can be either set to $15 \mathrm{kHz}$ or to 7.5 $\mathrm{kHz}$, whereas for normal CP it is $15 \mathrm{kHz}$ only.

It is worth highlighting that LTE provides robustness to a high extent against the detrimental effects induced by the user mobility. However, it is required that the Doppler shift is significantly lower than the subcarrier spacing and that the channel does not experience strong variations in one time slot. From the analysis conducted in Section III, it can be inferred that the aforementioned constraints will not be satisfied in the satellite systems under study. Consequently, the properties of primary and secondary synchronisation signals transmitted in the downlink frames could not be exploited to estimate the frequency misalignment between the transmitter and the receiver. In addition, reference signals embedded with the data cannot be used to estimate the channel with the desired granularity and, thus, channel variations cannot be tracked. In the light of this discussion, next section proposes solutions to overcome the impairments that are inherent to LEO satellite communication systems.

\section{B. Random Access}

In LTE, the RA procedure between UEs and RNs is the same as that between UEs and eNBs, [1], [13]. Two types of RA are present in LTE: i) contention-based, when the $\mathrm{UE}$ is not yet synchronised or lost its synchronisation; or ii) contention-free, in case the UE was previously synchronised to another eNB. Both procedures rely on the transmission of a random preamble from the UE to the eNB, [14]. Before it can start, the UE shall receive initial information from the broadcast control channels (e.g., the available set of resources for the RA). In Step 1, the UE randomly choses a preamble from a predefined set, which can also provide information on the expected amount of resources to be used, and sends it to the eNB along with a temporary network identifier. In Step 2, the eNB responds to the request with a RA Response (RAR) message, which shall be received by the UE within a RA time window between 3 and $15 \mathrm{~ms}$ after transmitting the preamble, [15]. In case the time expires, the UE can try again the procedure up to 200 times. Step 3 and 4 mainly aim at assigning to the UE a final network identifier and to resolve possible contentions. HARQ is used in Step 3, with a contention timer up to $64 \mathrm{~ms}$ and 1 to 8 tentatives. If the UE receives a correct response in Step 4, the procedure is successful and it is now logged to the LTE network. It is worthwhile highlighting that the contention-free procedure involves Step 1 and 2 only.

In the considered scenario, the UEs in each on-ground cell perform the RA procedure with the corresponding $\mathrm{RN}$, which terminates all protocols up to Layer 3. In particular, when implementing a contention-free RA, the only entities involved are the UE and the RN and, consequently, the delay on the satellite link is not involved and the procedure can be implemented without modifications. As for the contentionbased RA, in Step 3 and 4 the RN shall contact the EPC, through the DeNB, so as to obtain a final network identifier for the UE. In this moment, the delay on the satellite channel is involved and shall be carefully taken into account. However, as previously reported, the contention timer in this phase of the RA procedure is set to up to $64 \mathrm{~ms}$, which is much larger than the RTT between the RN and the EPC. Thus, no modifications are required in the LTE RA procedure in order to implement it over a LEO satellite system.

\section{RN attach procedure}

The RN attach procedure is performed in two steps, [1]: in the first phase, the RN performs a RA towards the DeNB as a UE so as to receive the permission and the parameters to operate as a relay, while in the second phase it attaches again to the DeNB specifically as a RN. After the start-up and attachment to the DeNB, the RN can start operating as a relay and serve the UEs in its cell.

In the considered scenario the RTT is in the order of 16 ms and in RA there are two timers to be taken into account: the RAR response window, with a duration up to $15 \mathrm{~ms}$, and the contention resolution timer, which can be fixed to up to $64 \mathrm{~ms}$. The latter does not introduce any technical difficulty, as it is much larger than the RTT. However, the RAR response window, i.e., the period in which the $\mathrm{RN}$ is expecting a response to its RA request, is actually lower than the RTT. Anyway, since this problem only arises at the RN start-up, the whole procedure can be replaced by ad-hoc network deployment. 


\section{Satellite handover}

In LTE, no mobility is foreseen for the RNs. However, in the considered system, the satellites in the LEO constellation move at great speed above the ground, i.e., while the RN and the DeNB are actually fixed, the satellite through which they communicate is not. In this context, we have two different effects depending on the layer that we are considering. On the one hand, at layers 2 and 3, the logical link between the RN and the DeNB is not aware of the satellite's movement and it shall remain active. On the other hand, the physical layer is deeply affected by the need to change the satellite that holds the connection with the DeNB, since a whole new PHY link must be established. This operation must be performed with a periodicity defined by the satellite visibility period, which is a function of its angular velocity and orbit.

\section{E. HARQ retransmission}

In the LTE MAC, up to 8 HARQ parallel processes are present in the HARQ entity and they are based on a Stop-AndWait (SAW) protocol, allowing to better exploit the available resources, [14]. Since the transmission is performed in $1 \mathrm{~ms}$, there is a $8 \mathrm{~ms}$ periodicity in the operations performed by each HARQ process. In the downlink, an adaptive (transmission attributes are adaptively reconfigured to the channel conditions) asynchronous (retransmissions can happen at any time, thus requiring an HARQ identifier) process is implemented. As for the uplink, the process is synchronous, thus not requiring the transmission of a HARQ identifier, and can be both adaptive or non-adaptive (the transmission parameters are modified on a predetermined basis). At the receiver side, soft combining with incremental redundancy is used. In the uplink, all (re-)transmissions must be triggered by a permission to be received $4 \mathrm{~ms}$ before and up to 28 retransmissions are allowed, [15]. The fixed $4 \mathrm{~ms}$ periodicity allows to reduce the signalling overhead, as no identifier is required at the receiver to understand the HARQ process to be involved. As for the downlink, the delay for retransmissions ranges from 4 to $7 \mathrm{~ms}$ and a proper HARQ identifier is required, being an asynchronous process. When taking into account RNs, due to the presence of broadcast signalling and synchronization signals, a $4 \mathrm{~ms}$ periodicity is not feasible in the uplink. In particular, the permission to retransmit the message, in some configurations, cannot be received $4 \mathrm{~ms}$ in advance. Thus, only configurations with a basic periodicity equal to $8 \mathrm{~ms}$ are implemented and up to 6 HARQ parallel processes are present, so as to minimize the delays, [16].

The critical aspect in analyzing LTE HARQ in the considered LEO system is thus the time in which the RN can retransmit the data and that in which it is expecting a feedback (ACK/NACK) from the DeNB. Based on the above observations, these two parameters are $8 \mathrm{~ms}$ and up to $7 \mathrm{~ms}$, respectively. As a consequence, it is not possible to use the standard HARQ procedure and proper solutions shall be found due to the large RTT over LEO satellites.

\section{Solutions}

In this section, we propose several alternatives for circumventing the challenges highlighted in Sec. IV.

\section{A. Waveform}

As already outlined in Sec. III, the Doppler shift on the backhaul link is increased by a factor $166<x<211$ with respect to the maximum Doppler experienced in traditional LTE and, thus, some modifications are required to implement it in the Sat-RN architecture. As a matter of facts, by equipping the RN with a GNSS receiver and providing also the trajectory of the satellite, then the Doppler could be compensated to a high extent. In case of an error in the estimation of the relative position between the $\mathrm{RN}$ and the satellite, a residual Doppler shift occurs. From geometrical considerations, the difference between the compensated and the actual Doppler shift can be computed. For the considered scenario and the carrier frequency in the Ku-band, and in order to keep the residual Doppler shift below $950 \mathrm{~Hz}$, it is possible to conclude that the position error must be smaller than $4 \mathrm{~km}$. In this case, there is no need to modify the LTE waveform.

The proof is as follows: Assume that $\mathbf{x}_{\mathrm{SAT}}(t)$ is known. Then, at a given time instant, the distance $|\mathbf{d}(t)|$ and the elevation angle $\theta(t)$ can be computed from (3) and (6). Knowing $\theta(t)$, the actual Doppler shift can be straightforwardly compensated according to (7). However, imagine that the assumed RN position is erroneous. Bearing in mind Fig. 4, the assumed Doppler would be computed from the duplet $\left(\left|\mathbf{d}_{e}(t)\right|, \theta_{e}(t)\right)$, which does not coincide with the real one, i.e., $(|\mathbf{d}(t)|, \theta(t))$. With reference to Fig. 4 , we can relate $\theta_{e}(t)$ to $R_{B}$, which defines the ambiguity region, and $(|\mathbf{d}(t)|, \theta(t))$ as

$$
\begin{gathered}
\left|\mathbf{d}_{e}(t)\right| \cos \left(\theta_{e}(t)\right)=|\mathbf{d}(t)| \cos (\theta(t))+R_{B} \\
|\mathbf{d}(t)|^{2}=\left|\mathbf{d}_{e}(t)\right|^{2}+R_{B}^{2}-2 R_{B}\left|\mathbf{d}_{e}(t)\right| \cos \left(\theta_{e}(t)\right)
\end{gathered}
$$

Solving (8), we end up with

$$
\cos \left(\theta_{e}(t)\right)=\frac{|\mathbf{d}(t)| \cos (\theta(t))+R_{B}}{\sqrt{|\mathbf{d}(t)|^{2}+R_{B}^{2}+2 R_{B}|\mathbf{d}(t)| \cos (\theta(t))}},
$$

which can be plugged into (7). By performing an exhaustive search for different values of $R_{B}$ and $45^{\circ} \leq \theta \leq 90^{\circ}$, it is possible to find the maximum position error that is tolerated such that the difference between the real and the assumed Doppler shift is below $950 \mathrm{~Hz}$.

\section{B. Satellite handover}

In order to cope with the satellites' movement, the RN shall be able to switch from the current satellite to another one in visibility as soon as the previous one falls behind the horizon. To this aim, two handover solutions can be identified:

1) PHY-based handover: since the logical link between the $\mathrm{RN}$ and the DeNB is not aware of the physical channel being used for the communication, the best solution would be that of performing a PHY handover, i.e., an handover procedure limited to the PHY, which does not affect the upper layers. 2) Traditional handover: if the complexity of implementing 


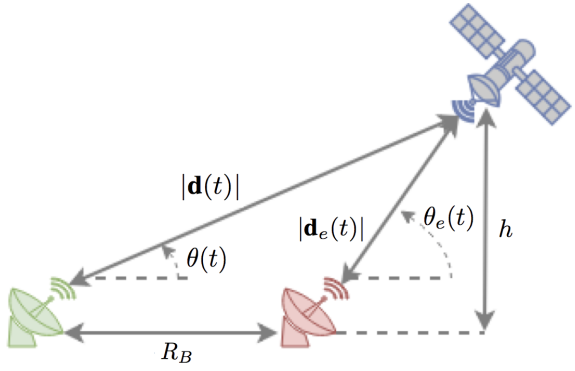

Fig. 4. RN position displacement.

a PHY-based handover is unacceptable, then the RN should perform a traditional handover. However, while complexity would be reduced, delays would be much larger. In particular, since for RNs the handover is not foreseen due to the absence of mobility, the attach procedure shall start from scratch again at each handover, thus significantly reducing the system flexibility and throughput as, after start-up, all of the UEs in the cell shall perform a new RA.

\section{C. $H A R Q$ retransmission}

In the HARQ procedure, the critical issue is that the 16 ms RTT is larger than both the time window in which the $\mathrm{RN}$ expects the ACK/NACK from the DeNB and the allowed retransmission instant. To cope with this challenge, three possible solutions can be identified:

1) Enhanced periodicity: similarly to the extended periodicity for RNs in the uplink described in Sec. IV.E, a possible solution might be that of extending the periodicity of the uplink HARQ retransmission by at least $16 \mathrm{~ms}$. As for the downlink, the time window in which the RN expects the DeNB feedback can be extended beyond $16 \mathrm{~ms}$, as well. However, this approach would deeply affect the overall throughput: since we are doubling the HARQ process periodicity, the throughput would be half that of the traditional LTE system as the retransmissions would be allowed after a doubled inactivity period, for a specific process.

2) Enhanced periodicity with reduced processes: the previous solution can be refined in order to maintain an acceptable throughput by reducing the number of parallel HARQ processes in the HARQ entity, similarly to the approach used to introduce RNs in LTE (see Sec. IV).

3) No $H A R Q$ with replication: in case extending the periodicity of the HARQ process is not acceptable due to throughput reduction, an alternative would be that of not implementing HARQ. In this case, in order to counteract the increased packet loss, the same packet can be replicated a certain amount of times at its transmission. This would indeed reduce the throughput as well, but with a much more limited impact.

In order to understand the performance of each of the proposed solution and, thus, clarify what type of modifications can be introduced in the HARQ procedure, a proper and extended throughput analysis is required.

\section{CONCLUSIONS}

In this paper, two architectures have been proposed for implementing a LTE-based LEO mega-constellation system. Focusing on the Sat-RN architecture, the impact of large Doppler shifts and delays has been discussed and assessed. On the backhaul link, the effect of the Doppler shifts on the waveform can be compensated by accurate location estimation and the RN attach procedure can be replaced by ad-hoc network deployment. However, the HARQ procedure requires a substantial modification since the RTT is twice the periodicity of the LTE procedures. Moreover, since relaying in LTE does not foresee mobility, a proper handover procedure shall be carefully designed in order to cope with the satellites' movement. Different solutions have been proposed for both the HARQ and the RN attach procedures, and their impact on the system throughput and complexity is left for further studies.

\section{ACKNOWLEDGMENT}

This work has been supported by European Space Agency (ESA) funded activity SatNEx IV COO1-PART 2 WI 7 "Air interface development for LEO constellation." The views of the authors of this paper do not reflect the views of ESA.

\section{REFERENCES}

[1] ETSI TS 136300 v13.4.0 (2016-08), LTE; Evolved Universal Terrestrial Radio Access (E-UTRA) and Evolved Universal Terrestrial Radio Access Network (E-UTRAN); Overall description; Stage 2, 2016.

[2] B. G. Evans, "The role of Satellites in 5G," 7th Adv. Sat. Mult. Syst. Conf. and the 13th Sign. Proc. for Sp. Comm. Work. (ASMS/SPSC), Sep. 2014.

[3] F. Bastia et al., "LTE Adaptation for Mobile Broadband Satellite Networks," EURASIP J. on Wir. Comm. and Net., vol. 2009, Mar. 2009.

[4] G. Araniti, M. Condoluci, and A. Petrolino, "Efficient Resource Allocation for Multicast Transmissions in Satellite-LTE Networks," IEEE Glob. Comm. Conf. (GLOBECOM), Dec. 2013.

[5] M. Amadeo, G. Araniti, A. Iera, and A. Molinaro, "A Satellite-LTE network with delay-tolerant capabilities: design and performance evaluation," IEEE Vehic. Tech. Conf. (VTC Fall), Sep. 2011.

[6] 3GPP TR 36.806 v9.0.0 (2010-03), 3rd Generation Partnership Project; Technical Specification Group Radio Access Network; Evolved Universal Terrestrial Radio Access (E-UTRA); Relay architectures for E-UTRA, 2010.

[7] ETSI TS 136116 v13.0.1 (2016-01), LTE; Evolved Universal Terrestrial Radio Access (E-UTRA); Relay radio transmission and reception, 2016.

[8] ETSI TS 136410 v13.0.0 (2016-01), LTE; Evolved Universal Terrestrial Radio Access Network (E-UTRAN); S1 general aspects and principles, 2016.

[9] ETSI TS 136411 v13.0.0 (2016-01), LTE; Evolved Universal Terrestrial Radio Access Network (E-UTRAN); S1 layer 1, 2016.

[10] I. Ali, N. Al-Dhahir, and J. E. Hershey, "Doppler characterization for LEO satellites," IEEE Trans. Commun., vol. 46, pp. 309-313, Mar. 1998.

[11] ETSI TS 136211 V13.2.0 (2016-08), LTE; Evolved Universal Terrestrial Radio Access (E-UTRA); Physical channels and modulation, 2016.

[12] ETSI TS 136213 V13.2.0 (2016-08), LTE; Evolved Universal Terrestrial Radio Access (E-UTRA); Physical layer procedures, 2016.

[13] ETSI TS 136216 v13.0.0 (2016-01), Universal Mobile Telecommunications System (UMTS); LTE; Evolved Universal Terrestrial Radio Access (E-UTRA); Physical layer for relaying operation, 2016.

[14] ETSI TS 136321 v13.3.0 (2016-10), LTE; Evolved Universal Terrestrial Radio Access (E-UTRA); Medium Access Control (MAC) protocol specification, 2016.

[15] ETSI TS 136331 v13.3.0 (2016-10), LTE; Evolved Universal Terrestrial Radio Access (E-UTRA); Radio Resource Control (RRC); Protocol specification, 2016.

[16] S. Sesia, I. Toufik, and M. Baker, LTE - The UMTS Long Term Evolution: From Theory to Practice, John Wiley \& Sons Inc Print on, 2nd ed., 2011. 